\title{
Cost and Utilization Comparisons Among Propensity Score-Matched Insulin Lispro and Regular Insulin Users
}

\author{
JENNIFER A. HALL, MPH; KENT H. SUMMERS, PhD; and ROBERT L. OBENCHAIN, PhD
}

\begin{abstract}
OBJECTIVE: To compare cost and utilization among users of insulin lispro and regular (human) insulin.

METHODS: This was a retrospective analysis using administrative claims data for continuously enrolled subjects using insulin lispro or regular insulin between January 1, 1998, and December 21, 1999. Subjects were matched 1 to 1 on the propensity to receive lispro versus regular insulin using a score estimated from baseline characteristics such as age, gender, comorbidities, and oral hypoglycemic use. Once matched, 12 months of follow-up pharmacy and medical cost and utilization data (e.g. prescriptions, office visits, hospitalizations) from July 1 , 1997, through December 31, 2000, were analyzed using univariate statistics.
\end{abstract}

RESULTS: Of 11,443 subjects, 3,341 (29.2\%) had received a prescription for insulin lispro, while $8,102(70.8 \%)$ had received a prescription for regular insulin. At baseline, lispro subjects tended to be younger, more often had type 1 diabetes and a history of insulin use, had fewer comorbidities, visited endocrinologists more often than family practice physicians, and had lower total costs. After matching on propensity score to within $\pm 0.01,1,832$ subject pairs were retained. On average, lispro subjects had significantly more office visits $(P=0.0022)$ and pharmacy prescriptions $(P=0.0165)$ but fewer inpatient hospital visits $(P=0.0028)$ compared to regular insulin subjects. Cost results were similar, with insulin lispro subjects having significantly higher average office visit costs $(P=0.0237)$ and pharmacy costs $(P<0.0001)$ but lower inpatient hospital costs $(P=0.0227)$. Total costs were not significantly different between treatment groups $(P=0.5266)$.

CONCLUSION: Total direct health care costs were not different between insulin lispro and regular insulin users. An association was observed between higher direct drug product cost and more intensive ambulatory care for insulin lispro users and lower inpatient hospital cost in the short-term.

KEYWORDS: Insulin, Lispro, Cost

J Managed Care Pharm. 2003(9)3: 263-68
$\mathrm{T}$ he average total charge for treating U.S. patients hospitalized for diabetes with complications increased from $\$ 10,271$ in 1993 to $\$ 14,779$ in 2000 . The total number of patients hospitalized increased from 373,666 to 455,027 during that time period. ${ }^{1}$ With diabetes treatment expenses and lost productivity reaching $\$ 98$ billion in the United States annually, the urgency for timely diagnosis, treatment, and better glycemic control continues. ${ }^{2}$

Advancements in the treatment of diabetes mellitus have long focused on improving glucose control. One such improvement has been the market availability of insulin lispro. Insulin lispro is a rapid-acting human insulin analog with a faster onset and shorter duration of action compared to regular human insulin. The shorter time to peak serum insulin level more closely mimics physiologic secretion of insulin, which results in greater relative reductions of postprandial blood glucose concentrations. Better clinical outcomes (superior postprandial glycemic control without an increase in the risk of severe hypoglycemia) with insulin lispro have been demonstrated in numerous clinical trials. ${ }^{3-8}$ There are, however, few economic studies of insulin lispro use. ${ }^{9,10}$ While these studies demonstrated that consumers perceive the benefits of insulin lispro therapy to justify its additional cost relative to regular (human) insulin, no studies have been published that examine whether insulin lispro's higher drug cost is offset by direct medical cost savings in areas of the health care system outside of pharmacy.

The objective of this study was to assess potential economic benefits of insulin lispro use compared to regular insulin in a population of managed care enrollees with diabetes. This study was designed to address questions from the perspective of the employer, who has the option to choose among a variety of benefit designs, i.e., which drugs to cover at a preferred status versus a nonpreferred status. An analysis that considers both thirdparty payer payments and patient copayments is relevant when considering the true cost of insulin. This study addressed the question of whether the higher total product cost of insulin lispro compared to regular insulin therapy is offset by health care cost savings in nonpharmacy areas.

\section{Methods}

Ingenix, Eden Prairie, Minnesota; KENT H. SUMMERS, PhD, is a Research Scientist, and ROBERT L. OBENCHAIN, PhD, is a Senior Research Scientist, Eli Lilly \& Company, Indianapolis, Indiana.

AUTHOR CORRESPONDENCE: Jennifer A. Hall, MPH, Researcher, Economic and Outcomes Division, Ingenix, 12125 Technology Dr., MN002-0258, Eden Prairie, MN 55344. Tel: (952) 833-8310; Fax: (952) 833-6045;

E-mail: jennifer.hall@ingenix.com

Copyright $\odot$ 2003, Academy of Managed Care Pharmacy. All rights reserved.

\section{Study Site and Data Source}

This study was conducted using enrollment, medical, and pharmacy claims data from 14 UnitedHealthcare affiliated health plans located throughout the southeastern, northeastern, midwestern, and western United States. These health plans are independent practice association model plans that employed the discounted, fee-for-service method for provider reimbursement. 
APPENDIX A Baseline Variables Used in Logistic Model to Derive Propensity Scores

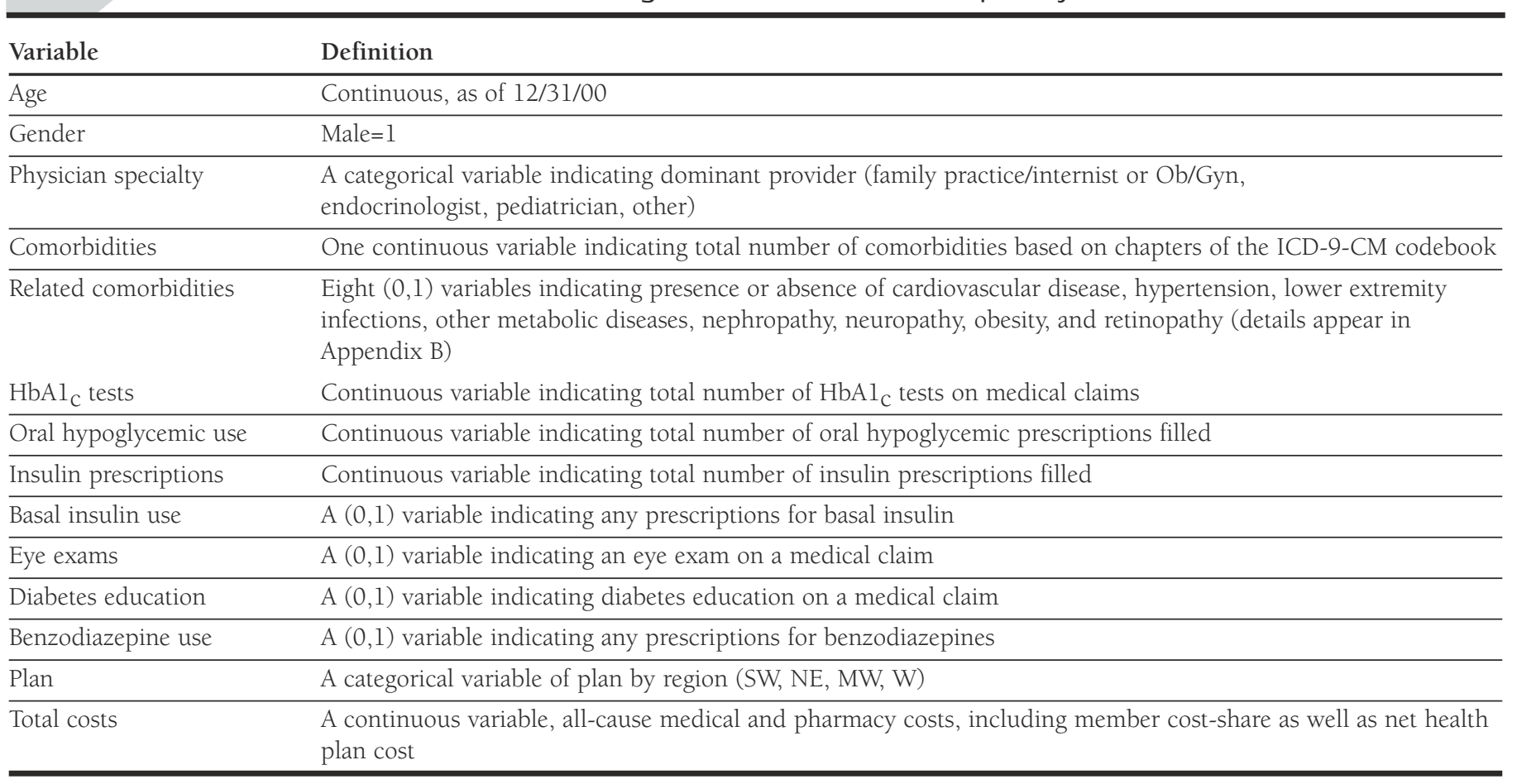

\section{Study Period}

All prevalent users of insulin from January 1, 1998, through December 31, 1999, were identified in the claims database. For each subject, the fill date of the first insulin prescription during that 24-month period was considered the study index date. Each subject's health services utilization patterns were examined for a period from 6 months prior through 12 months following this study index date. Thus, the study included pharmacy and medical claims data from July 1, 1997, through December 31, 2000.

\section{Inclusion Criteria}

Health plan enrollees meeting all of the following inclusion criteria were selected as study subjects: (1) at least 1 pharmacy claim for insulin lispro or regular insulin between January 1 , 1998, and December 31, 1999; (2) continuous enrollment for at least 6 months prior to and 12 months following the study index date; and (3) drug benefit coverage during the entire 18-month continuous enrollment period.

\section{Treatment Groups}

If a subject filled a prescription for insulin lispro between January 1, 1998, and December 31, 1999, the subject was included in the lispro group. If a regular insulin prescription was filled and no insulin lispro prescription was filled in this time frame, the subject was included in the regular insulin group.

\section{Propensity Score Matching}

Subjects were matched on the propensity to receive lispro insulin versus regular insulin. Matching subjects on propensity scores is one method of controlling for confounding when numerous characteristics are related to the outcome of interest or when 2 populations are known to differ due to selection bias. This method serves to balance the treatment groups at baseline. ${ }^{11}$ While standard regression modeling can handle several regressor variables, results can be misleading because small differences in numerous covariates can accumulate into a substantial overall difference. Two treatment groups may differ in a multivariate direction to an extent that cannot be discerned in the separate analyses of each covariate. ${ }^{12}$ For this reason, propensity score methodology is a reasonable alternative. In this study, baseline characteristics were used as independent predictors in a multivariate logistic regression model. The model was constructed to predict the probability (score) of receiving lispro versus regular insulin. Subjects were subsequently matched ( 1 to 1 ) on propensity scores within \pm-0.01 . Subjects who could not be matched were removed from further analysis. Baseline characteristics were compared before and after matching to insure that all significant differences between the treatment groups had become nonsignificant. The independent variables used in the logistic regression model are defined in Appendix A. Due to the large number of comparison tests $(\mathrm{N}=21)$, the alpha level for all comparison tests was adjusted using a Bonferroni correction procedure, which resulted in 
TABLE 1 Bivariate Tests Before and After Matching Lispro and Regular Insulin Subjects

\begin{tabular}{lcc}
\hline & $\begin{array}{c}P \text { Value } \\
\text { Before Matching } \\
\text { Lispro N=4,518 } \\
\text { Regular N=11,155 }\end{array}$ & $\begin{array}{c}\text { P Value } \\
\text { After Matching } \\
\text { Lispro N=1,832 } \\
\text { Regular N=1,832 }\end{array}$ \\
Variable & $<0.0001$ & 0.7121 \\
\hline Basal insulin use & 0.0068 & 0.8268 \\
\hline Diabetes education & $<0.0001$ & 0.2445 \\
\hline Eye exam & $<0.0001$ & 0.6746 \\
\hline Benzodiazepine use & $<0.0001$ & 0.3902 \\
\hline Age (in years) & $<0.0001$ & 0.7764 \\
\hline Dominant physician specialty & $<0.0001$ & 0.8866 \\
\hline Number of baseline HbAl C tests & $<0.0001$ & 0.9399 \\
\hline Number of baseline insulin prescriptions & $<0352$ & 0.3467 \\
\hline Health plan location & $<0.0001$ & 0.6957 \\
\hline Number of baseline comorbidities & $<0.0001$ & 0.8919 \\
\hline Cardiovascular disease in baseline & $<0.0001$ & 0.5032 \\
\hline Hypertension in baseline & $<0.0001$ & 0.4431 \\
\hline Infection in baseline & 0.0708 & 0.8645 \\
\hline Metabolic disease in baseline & 0.1107 & 0.7189 \\
\hline Nephropathy in baseline & 0.9233 & 0.7350 \\
\hline Neuropathy in baseline & $<0.0001$ & 0.4127 \\
\hline Obesity in baseline & 0.0011 & 0.3221 \\
\hline Retinopathy in baseline & 0.0436 & 0.3903 \\
\hline Gender (male=1) & $<0.0001$ & 0.0001 \\
\hline Total baseline costs & & \\
\hline Oral hypoglycemic use* & & \\
\hline * Based on an algorithm combining diagnosis codes and presencelabsence of oral \\
hypoglycemics, 14.9\% of lispro and $8.8 \%$ of regular insulin subjects appeared to \\
have type 1 diabetes prior to matching compared to 11\% and 10.4\% after \\
matching, respectively.
\end{tabular}

using a corrected alpha of 0.0024

\section{Measuring Follow-up Cost and Utilization}

Health services utilization and costs were analyzed by type of service (physician office visit, outpatient hospital visit, inpatient hospitalization, emergency room visit, pharmacy, and laboratory/radiology tests). For the purpose of this study, costs were defined as the sum of both health plan and enrollee liability; i.e., total allowed managed care organization charges before subtraction of member-cost share. $T$ tests with an alpha level of 0.05 were used to detect significant differences between treatment groups with regard to follow-up cost and utilization.

\section{Results}

\section{Study Population Selection}

A total of 29,878 subjects had at least 1 insulin claim during the study identification period (January 1, 1998, through December $3,1999)$, corresponding to a prevalence of 7 insulin-treated enrollees per 1,000 health plan enrollees. From this population, 11,443 subjects met the study inclusion criteria: 3,341 (29.2\%) insulin lispro subjects and 8,102 (70.8\%) regular insulin subjects. Of those excluded from study eligibility, 15,200 (82\%) were dropped due to lack of continuous enrollment, while 3,235 (18\%) were excluded due to receipt of an insulin prescription that was neither insulin lispro nor regular insulin. The majority of these 3,235 subjects had prescriptions filled for $\mathrm{NPH}$, an intermediate-acting insulin often used in concert with either insulin lispro or regular insulin.

\section{Summary of Matching Procedure}

Prior to matching subjects by their propensity scores (predicted value of receiving insulin lispro versus regular insulin based on baseline characteristics), 21 different baseline characteristics were compared across the 2 treatment groups. For 15 of the 21 (71.4\%) baseline characteristics, the 2 treatment groups differed significantly. Insulin lispro subjects tended to be younger, used fewer oral hypoglycemics, had fewer comorbidities, visited endocrinologists more often than family practitioners, and had lower total costs compared to subjects who received regular insulin (Table 1). Of 3,341 insulin lispro subjects, 1,832 (54.8\%) subjects were matched to a regular insulin-using subject within \pm-0.01 . All subsequent analyses were limited to this matched sample ( $\mathrm{N}=3,664$ subjects [ 1,832 subject pairs]). After matching, none of the 21 baseline comparison tests remained significantly different. Characteristics of subjects lost during the matching procedure are summarized below.

The lispro subjects who were not matched were more often type 1 diabetics (based on an algorithm combining ICD-9-CM diagnosis codes [250.xx, Appendix B] and presence or absence of prescriptions for oral hypoglycemic agents) who were younger; prevalent users of insulin; treated by specialists; had less circulatory disease, cardiovascular disease, hypertension, or obesity; filled more insulin prescriptions; and had more $\mathrm{HbAl}_{\mathrm{C}}$ tests compared to the lispro subjects who were matched. The regular insulin subjects for whom no match existed were more often older; were treated by general practitioners; had more neoplasms and circulatory, digestive, cardiovascular and musculoskeletal disease, hypertension, ill-defined conditions, lower extremity infections, and obesity; had fewer pregnancy complications, $\mathrm{HbAl}_{\mathrm{C}}$ tests, and insulin prescriptions; had more oral hypoglycemic prescriptions; and had higher baseline pharmacy and total costs.

\section{Follow-up Cost and Utilization Analysis}

Health services utilization during the 12-month follow-up period was compared across the 2 treatment groups (Table 2). While average rates of outpatient hospital visits, emergency room visits, or lab tests did not differ significantly between the 2 treatment groups, there were significant differences detected in numbers of office visits, prescriptions filled, and inpatient hospitalizations. On average, insulin lispro subjects had signifi- 


\section{APPENDIX B ICD-9-CM Codes for Comorbidities Related to Complications of Diabetes}

\begin{tabular}{ll}
\hline Comorbidity/Complication & ICD-9-CM Diagnosis Codes and CPT Codes \\
\hline Cardiovascular disease & $410 . x x-414 . x x, 415 . x x-417 . x x, 420 . x x-429 . x x, 430 . x x-438 . x x, 440 . x x-448 . x x, 785.4 x$ \\
\hline Hypertension & $401 . x x-404 . x x$ \\
\hline Infections related to diabetes & $038 . x x, 790.7 x-790.9 x$ \\
\hline Other metabolic diseases & $251.0 x-251.3 x, 270.3 x, 276 . x x$ \\
\hline Nephropathy & $583.81,580.9 x, 581.81,581.9 x, 582.9 x, 583 . x x, 588.8 x, 593.9 x$ \\
\hline Neuropathy & $358.01,354 . x x-355 . x x, 713.5 x, 337.1 x, 357.2 x$ \\
\hline Obesity & $278 . x x$ \\
\hline Retinopathy & $362.0 x, 362.1 x, 362.2,362.41,363.31,369 . x x, 366.41,365.44$ \\
\hline
\end{tabular}

\section{TABLE 2 Univariate Comparison of All-Cause Health Services Utilization During Follow-up Period} on Propensity Score-Matched Subjects

\begin{tabular}{|c|c|c|c|c|c|}
\hline Office visits & 1,777 & 1,754 & 11.68 & 10.60 & 0.0022 \\
\hline Emergency room & 524 & 546 & 0.51 & 0.52 & 0.6742 \\
\hline Outpatient hospital & 1,114 & 1,051 & 2.02 & 2.48 & 0.0712 \\
\hline Inpatient hospitalization & 290 & 388 & 0.24 & 0.32 & 0.0028 \\
\hline Laboratory tests & 1,505 & 1,483 & 4.70 & 4.40 & 0.1692 \\
\hline Pharmacy & 1,826 & 1,821 & 47.16 & 44.44 & 0.0165 \\
\hline
\end{tabular}

cantly more office visits ( $P=0.0022)$ and filled significantly more prescriptions (diabetes-related and nondiabetes-related prescriptions, $P=0.0165$ ) compared to regular insulin subjects. In contrast, insulin lispro subjects had, on average, significantly fewer inpatient hospitalizations compared to regular insulin subjects $(P=0.0028)$. Among subjects who received at least one diagnosis of hypoglycemia in the follow-up period, insulin lispro subjects had a significantly lower average number of hypoglycemia-related hospitalizations $(P=0.0014)$.

Cost during the 12-month follow-up period was compared across the 2 treatment groups (Table 3). Insulin lispro subjects had significantly higher average office visit costs and pharmacy costs compared to regular insulin subjects $(P=0.0237$ and $P<0.0001$, respectively) as well as significantly lower average inpatient hospital costs compared to regular insulin subjects $(P=0.0227)$; there was no significant difference in average emergency room, outpatient, laboratory, or total costs. It is important to note that although lispro subjects did have significantly higher average office visit costs and pharmacy costs (+\$106 and $+\$ 447$, respectively) relative to regular insulin subjects, these higher costs were offset by lower average inpatient hospital cost
(-\$769), a cost savings for insulin lispro (albeit not statistically significant) of \$216 during the 12-month follow-up period.

\section{Discussion}

With its faster onset and shorter duration of action compared to regular insulin, insulin lispro has demonstrated a decreased risk of severe hypoglycemia compared to regular insulin.-8 Type 1 patients taking insulin lispro also report improved satisfaction with their treatment and its flexibility. ${ }^{13-17}$ This study sought to determine whether the use of insulin lispro would result in no additional health care costs (cost neutral) as compared to regular insulin therapy.

As anticipated, subjects at baseline who were treated with insulin lispro differed significantly from those treated with regular insulin. Insulin lispro subjects tended to be younger, use fewer oral hypoglycemics, were less likely to be a new insulin user, were more likely to be treated by an endocrinologist or pediatrician, had fewer comorbidities, received more preventive care (e.g. eye exams, diabetes education, $\mathrm{HbAl}_{\mathrm{c}}$ tests), and had fewer inpatient visits, pharmacy prescriptions, or laboratory tests during the 6 months prior to the study period as compared 


\begin{tabular}{|c|c|c|c|}
\hline \multicolumn{4}{|c|}{ Mean cost } \\
\hline Type of Cost & Lispro & Regular & $P$ Value* \\
\hline Office visit & $\$ 822$ & $\$ 716$ & 0.0237 \\
\hline Emergency visit & $\$ 185$ & $\$ 177$ & 0.6433 \\
\hline Outpatient hospital & $\$ 1,008$ & $\$ 1,062$ & 0.7077 \\
\hline Inpatient hospital & $\$ 1,741$ & $\$ 2,510$ & 0.0277 \\
\hline Laboratory & $\$ 233$ & $\$ 251$ & 0.4200 \\
\hline Pharmacy & $\$ 2,244$ & $\$ 1,797$ & $<0.0001$ \\
\hline Total & $\$ 6,231$ & $\$ 6,511$ & 0.5266 \\
\hline Total PPPM $\dagger$ & $\$ 519$ & $\$ 543$ & 0.5266 \\
\hline
\end{tabular}

to regular insulin users.

After propensity score procedures were completed, insulin lispro subjects were found to have significantly higher expenditures for office visits and prescription drug use compared to their regular insulin matches, while having significantly lower inpatient hospitalization expenditures. The significantly higher pharmacy expenditures found in the insulin lispro group were not surprising because the direct product cost of insulin lispro is greater than for regular insulin. In addition, lispro users had more prescriptions for blood glucose monitoring devices and other insulin supplies. The significantly higher office visit expenditures for patients using insulin lispro may be a function of those subjects being more conscientious about their followup care. Because insulin lispro is often used in combination with other longer-acting insulins, diabetes patients sufficiently vigilant to comply with such types of dual therapy may also be more vigilant regarding follow-up office visits.

This study did yield a result that indicates an association between increased office visit and pharmacy expenditures and lower inpatient hospital expenditures incurred by insulin lispro subjects over the short-term, 12-month follow-up period. This observation may indicate that insulin lispro's flexibility with regard to dosing and timing of meals led to fewer incidents of severe hypoglycemia, which could potentially result in an inpatient hospitalization, especially within a population of type 1 patients. ${ }^{18,19}$ In fact, among subjects who received a diagnosis of hypoglycemia during the follow-up period, insulin lispro subjects had significantly fewer hypoglycemia-related inpatient hospitalizations. Cost savings associated with fewer hospitalizations for insulin lispro patients may go beyond direct health care costs to include, for example, lower indirect costs associated with lost workdays due to hospitalization. The results observed here support our supposition that, when considering total medical costs, the utilization of insulin lispro could be cost neutral to an employer in an administrative services-only arrangement with health maintenance or preferred provider organization plans. Further research is necessary to determine whether the result of fewer hospitalizations would be corroborated over a longer follow-up period.

\section{Limitations}

While this study faced some limitations, we believe these limitations did not compromise the overall study findings. The following limitations should be observed when interpreting the study results. First, subjects were categorized as "insulin lispro" users if they had at least 1 lispro prescription during the subject identification period. Therefore, a subject could have switched therapy during the study period. However, $<1 \%$ of regular insulin users filled a lispro prescription during the follow-up period, suggesting that alternative therapy did not attribute to outcomes observed during the follow-up period. Second, compliance with therapy was not measured and could account for some of the differences in outcomes. Third, because the 2 populations of subjects were quite different at baseline, the matching technique likely resulted in the pairing of a "sicker" lispro subject and a "healthier" regular insulin subject at baseline. However, this method also had the advantage of taking away much of the "noise" that would cloud true associations when starting with 2 populations that were very different. Fourth, the study design included prevalent insulin users. While prior insulin use was controlled for in the propensity score-matching procedure, it may be preferable to include only new users of insulin in future studies. Due to the relatively small number of lispro insulin subjects, both prevalent and incident insulin users were retained for study as a way to preserve sample size. Fifth, while propensity score matching can control for selection bias, it can only control for known or measurable confounders. As with many statistical techniques, residual confounding was still a possibility. Finally, this study used only 12 months of follow-up data and claims through December 31, 2000. It would be beneficial to repeat this study with more current data and also allow for a longer period of follow-up time. Studies such as the United Kingdom Prospective Diabetes Study and the Diabetes Control and Complications Trial determined patient outcomes for an average of 10 and 7 years, respectively.20,21

\section{Future Research}

Based on the study results, there are several possibilities for future research. First, further studies could examine only diabetes-related costs and utilization, as opposed to all-cause costs and utilization studied here, to determine whether the above relationships remain similar. Second, results could be examined separately for pediatric and adult populations. Differences in utilization patterns by treatment type may vary even within the pediatric population; the benefit of insulin lispro's flexibility with regard to dosing and timing of meals may be far more beneficial in teenagers, a population prone to forgotten or otherwise 
missed doses, as compared to children under 6 whose dosing may be closely supervised by a parent. Third, results could be stratified according to type 1 or type 2 diabetes status because of the differences in comorbidities and demographic characteristics, qualities that may influence their health-seeking behavior and treatment outcomes. Fourth, a longer follow-up period may better illuminate treatment differences particularly with regard to hospitalization. Continuous enrollment requirements for retrospective database studies often limit sample size. However, results could be reported for each subset of subjects enrolled 12 months, 24 months, 36 months, etc. Finally, the addition of laboratory data, such as $\mathrm{HbAl}_{\mathrm{C}}$ tests, may better explain why insulin lispro users had significantly less hospital utilization as compared to regular insulin users.

\section{Conclusion}

This study aimed to show that despite its higher total product cost, use of insulin lispro would be associated with total direct medical care costs similar to regular (human) insulin therapy. Findings from this study supported this supposition. We observed lower inpatient hospital expenditures during the 12-month study period, which appeared to offset the higher cost attributable to more intensive ambulatory patient care and the higher direct drug cost of lispro insulin. These results should be weighed by managed care organizations in the context of prior evidence from clinical trials of lower risk of severe hypoglycemia and dosing flexibility for patients who use insulin lispro.

\section{ACKNOWLEDGMENTS}

The authors would like to thank Pam Erickson, MS; John Holcombe, MD; and Scott Jacober, DO, CDE, for their helpful reviews of this paper.

\section{DISCLOSURES}

Funding for this study was provided by Eli Lilly \& Company and was obtained by author Kent H. Summers, who is employed by Lilly. Author Robert L. Obenchain is also a Lilly employee and author of papers proposing and comparing alternative methods of propensity scoring. Author Jennifer A. Hall is an employee of Ingenix, which was contracted by Eli Lilly \& Company to complete the research. Hall served as principal author of the study and was responsible for the analysis and interpretation of data and drafting of the manuscript. Study concept and design and critical revision of the manuscript were the work of Summers and Obenchain. Statistical expertise was contributed by Obenchain

An abstract of this research was printed in Value in Health following a poster presentation at the May 2002 Annual International ISPOR meeting.

\section{REFERENCES}

1. Agency for Healthcare Research and Quality. Healthcare Cost and Utilization Project. HCUPnet, 2000. Available at:

www.ahrq.gov/data/hcup/hcupnet.htm.

2. American Diabetes Association. Facts and Figures. Available at: www.diabetes.org/ada/facts.asp.

3. Schernthaner G, Wein W, Sandholzer K, et al. Postprandial insulin lispro. A new therapeutic option for type 1 diabetic patients. Diabetes Care. 1998;21(4):570-73.

4. Toth EL, Lee KC. Guidelines for using insulin lispro. Can Fam Physician. $1998 ; 44: 2444-49$

5. Roach P, Yue L, Arora V. Improved postprandial glycemic control during treatment with Humalog Mix 25, a novel protamine-based insulin lispro formulation. Diabetes Care. 1999;22(8):1258-61.

6. Raskin P, Holcombe JH, Tamborlane WV, et al. A comparison of insulin lispro and buffered regular human insulin administered via continuous subcutaneous insulin infusion pump. J Diabetes Complications. 2001;15(6):295-300.

7. Deeb LC, Holcombe JH, Brunelle R, et al. Insulin lispro lowers postprandial glucose in prepubetal children with diabetes. Pediatrics. 2001;108(5):1175-79.

8. Ferguson SC, Strachan MW, Janes JM, Frier BM. Severe hypoglycaemia in patients with type 1 diabetes and impaired awareness of hypoglycaemia: a comparative study of insulin lispro and regular human insulin. Diabetes Metab Res Rev. 2001;17(4):285-91.

9. Davey P, Grainger D, MacMillan J, et al. Economic evaluation of insulin lispro versus neutral (regular) insulin therapy using a willingness-to-pay approach. Pharmacoeconomics. 1998;13(3):347-58.

10. Dranitsaris G, Longo CJ, Grossman LD. The economic value of a new insulin preparation, Humalog Mix 25. Measure by a willingness-to-pay approach. Pharmacoeconomics. 2000;18(3):275-87.

11. D'Agostino RB Jr. Tutorial inbiostatistics: propensity score methods for bias reduction in the comparison of a treatment to a non-randomized control group. Stat Med. 1998;17:2265-81.

12. Rubin DB. Estimating causal effects from large data sets using propensity scores. Ann Intern Med. 1997;127:757-63.

13. Valle D, Santoro D, Bates P, Scarpa L. Italian multi-center study of intensive therapy with insulin lispro in 1184 patients with type 1 diabetes. Diabetes Nutr Metab. 2001;14(3):126-32

14. Kotsanos JG, Vignati L, Huster W, et al. Health-related quality-of-life results from multinational clinical trials of insulin lispro. Diabetes Care. 1997;20(6):948-58.

15. Grey M, Boland EA, Tamborlane WV. Use of lispro insulin and quality of life in adolescents on intensive therapy. Diabetes Educ. 1999;25(6):934-41.

16. Holleman F, Schmitt H, Rottiers R, et al. Reduced frequency of severe hypoglycemia and coma in well-controlled IDDM patients treated with insulin lispro. Diabetes Care. 1997;20(12):1827-32

17. Howorka K, Pumprla J, Schlusche C, et al. Dealing with ceiling baseline treatment satisfaction level in patients with diabetes under flexible, functional insulin treatment: assessment of improvements in treatment satisfaction with a new insulin analogue. Qual Life Res. 2000;9(8):915-30.

18. Brunelle BL, Llewelyn J, Anderson JH Jr, Gale EA, Koivisto VA. Metaanalysis of the effect of insulin lispro on severe hypoglycemia in patients with type 1 diabetes. Diabetes Care. 1998;21:1726-31.

19. Holleman F, Schmitt H, Rottiers R, Rees A, Symanowski S, Anderson JH Reduced frequency of severe hypoglycemia and coma in well-controlled IDDM patients treated with insulin lispro. The Benelux-UK Insulin Lispro Study Group. Diabetes Care. 1997; 20:1827-32

20. American Diabetes Association. Implications of the Diabetes Control and Complications Trial. Diabetes Care. 2003;26(suppl 1):S25-S27.

21. American Diabetes Association. Implications of the United Kingdom Prospective Diabetes Study. Diabetes Care. 2003;26(suppl 1):S28-S32. 